\title{
(2) OPEN ACCESS \\ Implementing an early rule-out pathway for acute myocardial infarction in clinical practice
}

\author{
Matthew T H Lowry (ㄷ, , Atul Anand 지, ${ }^{1}$ Nicholas L Mills (1) 1,2
}

${ }^{1}$ BHF Centre for Cardiovascular Science, The University of Edinburgh, Edinburgh, UK 2Usher Institute, The University of Edinburgh, Edinburgh, UK

\section{Correspondence to}

Dr Nicholas L Mills, BHF Centre for Cardiovascular Sciences, University of Edinburgh Division of Clinical and Surgical Sciences, Edinburgh EH8 9YL, UK; nick.mills@ed.ac.uk

Published Online First 3 September 2021

Check for updates

(C) Author(s) (or their employer(s)) 2021. Re-use permitted under CC BY-NC. No commercial re-use. See rights and permissions. Published by BMJ.

To cite: Lowry MTH Anand A, Mills NL. Heart 2021:107:1912-1919.

\section{INTRODUCTION}

Chest pain is a common presentation to the emergency department and can be caused by a range of conditions including acute myocardial infarction. However, only 1 in 10 patients with symptoms suggestive of acute coronary syndrome are ultimately diagnosed with myocardial infarction. As such, effective pathways are required to enable the prompt and safe rule-out of the majority of patients with non-cardiac presentations and the rapid identification of those with myocardial infarction.

Recently published guidelines from the National Institute for Health and Care Excellence (NICE) and the European Society of Cardiology (ESC) have recommended the use of early rule-out pathways for myocardial infarction, ${ }^{23}$ enabled by the increased analytical precision of high-sensitivity cardiac troponin (hs-cTn) testing. ${ }^{4}$ These guideline recommendations are supported by recent randomised trials that have provided new insights into the safety and effectiveness of these pathways in clinical practice..$^{5-7}$ Multiple pathways have been proposed that vary according to the thresholds used for decision-making and timing of sampling. Implementing a validated pathway could save healthcare resources and improve the safe delivery of patient care.

Here we describe these early rule-out pathways, review their supporting evidence and provide practical advice for their adoption in clinical practice.

\section{DIAGNOSTIC THRESHOLDS FOR ACUTE MYOCARDIAL INFARCTION}

Cardiac troponin is a highly specific marker of cardiomyocyte injury, which can be detected in the circulation within an hour of the onset of myocardial ischaemia. ${ }^{8}$ High-sensitivity assays have sufficient analytical precision to quantify very low concentrations of cardiac troponin in the majority of healthy people. ${ }^{9}$ The 99 th centile upper reference limit is defined as the troponin concentration below which $99 \%$ of a healthy reference population are observed. The 99th centile differs for all cardiac troponin assays as no standardisation has been performed between platforms or manufacturers. Contemporary sensitive assays are unable to measure troponin within the reference range, and as a consequence cannot be used in any of the early rule-out pathways that use thresholds below the 99th centile to guide clinical decisions. It is essential when considering the adoption of an early rule-out pathway to understand the assay used in the hospital.
Learning objectives

- To understand the evidence and guideline recommendations for use of high-sensitivity cardiac troponin with early rule-out pathways for myocardial infarction.

- To recognise the strengths and limitations of different pathways including the clinical situations in which they are not suitable.

- To understand how to implement an early ruleout pathway in clinical practice.

\section{DEFINITION OF MYOCARDIAL INFARCTION}

The Fourth Universal Definition of Myocardial Infarction is a consensus statement endorsed by the world's major cardiovascular associations. ${ }^{10}$ Myocardial infarction is defined in those with symptoms or signs of myocardial ischaemia who have a rise and/or fall in cardiac troponin concentration, with at least one measurement above the 99th centile. ${ }^{10}$ hs-cTn assays are recommended, and given that the 99th centile differs in men and women for all troponin assays ${ }^{11-13}$ sex-specific thresholds are recommended to ensure equity of care. ${ }^{10}$ The 99 th centile is also influenced by age and presence of comorbidities, such as renal and heart failure, and this should be considered in patients with chronically elevated cardiac troponin concentrations. ${ }^{14} 15$

The universal definition of myocardial infarction recognises five subtypes based on the underlying mechanism and clinical situation in which myocardial infarction occurs (table 1). ${ }^{10}$ Given the majority of these events are due to systemic or non-coronary conditions or arise in specific clinical situations, studies evaluating the performance of early rule-out pathways have focused on type 1 myocardial infarction. ${ }^{67}$ Patients presenting with a systemic condition that results in major haemodynamic compromise are unlikely to be considered for early discharge whether or not they have secondary myocardial ischaemia.

\section{OPTIMISED RULE-OUT THRESHOLDS FOR ACUTE MYOCARDIAL INFARCTION}

Cardiac troponin is released rapidly into the circulation in response to myocardial ischaemia. ${ }^{8}$ Although increases are measurable within 1 or 2 hours of symptom onset, they peak 10-12 hours later and may not cross the 99th centile at early time points (figure 1). As such, it is not possible to safely rule out myocardial infarction using the 99th centile at presentation in patients presenting within 6 hours of symptom onset. Given this represents 
Table 1 Classification of myocardial infarction

\begin{tabular}{ll}
\hline Classification & Mechanism \\
\hline Type 1 & Acute atherothrombotic plaque rupture or erosion. \\
Type 2 & $\begin{array}{l}\text { A reduction in myocardial oxygen supply (eg, hypotension, hypoxia, anaemia) or } \\
\text { an increase in demand (eg, tachyarrhythmia) without atherothrombosis. }\end{array}$ \\
Type 3 & $\begin{array}{l}\text { Cardiac death prior to biomarker sampling or myocardial infarction detected on } \\
\text { autopsy. }\end{array}$ \\
Type 4a-c & Myocardial infarction associated with percutaneous coronary intervention. \\
Type 5 & Myocardial infarction associated with coronary artery bypass grafting. \\
\hline
\end{tabular}

the majority of patients with suspected acute coronary syndrome, researchers have sought to identify alternative thresholds that harness the ability of hs-cTn assays to measure very low cardiac troponin levels within the reference range. By using separate rule-out and rule-in thresholds, the diagnosis of myocardial infarction can be confidently excluded in more patients with a single test.

The optimal thresholds to rule out myocardial infarction for both hs-cTnI and hs-cTnT assays were derived in observational cohort studies and have since been validated in prospective implementation studies (figure 2). ${ }^{5-7} 1617$ The rule-out threshold should balance safety and effectiveness and has been proposed as the highest cardiac troponin concentration that achieves a negative predictive value of $99.5 \%$ for myocardial infarction, in order to maximise the proportion of patients who can be safely ruled out using a single test . For most hs-cTnI assays a threshold of less than $5 \mathrm{ng} / \mathrm{L}$ identifies half of all patients with suspected acute coronary syndrome as low risk with a false negative rate of less than 1 in 200. This group of patients are also at very low risk of cardiovascular events up to 1 year later. ${ }^{16} 18$ This rule-out threshold has been prospectively validated and the negative predictive value is consistently $>99.5 \%$ in both sexes and across all age groups. ${ }^{19}$

For the hs-cTnT assay the threshold with optimal diagnostic performance to rule out myocardial infarction is equivalent to the limit of detection of the assay (also less than $5 \mathrm{ng} / \mathrm{L}$ ), which represents the lowest measurable concentration in a sample. The limit of detection varies between hs-cTnI assays $(\sim 2 \mathrm{ng} / \mathrm{L})$, but it is clear from multiple observational studies that patients with troponin concentrations below this limit are at very low risk of future cardiac events. ${ }^{172021}$ However, the performance of the limit of detection as a decision threshold varies by platform and reagent batch, and for some assays with very high precision at low concentrations the proportion of patients with undetectable cardiac troponin is small, reducing the effectiveness of this approach. ${ }^{18} 22$ The safety and efficacy of a rule-out threshold of $5 \mathrm{ng} / \mathrm{L}$ were compared with the limit of detection of $2 \mathrm{ng} / \mathrm{L}$ in 32837 consecutive patients with suspected acute coronary syndrome across 10 hospitals. ${ }^{19}$ The rule-out threshold identified twice as many patients as low risk compared with the limit of detection at presentation $(71 \%$ vs $39 \%)$, with a similar and negative predictive value $(99.8 \%$ vs $99.9 \%)$.

\section{INTERMEDIATE HS-CTN CONCENTRATIONS}

The use of separate rule-out and diagnostic thresholds identifies a population of patients with intermediate cardiac troponin values (figure 2). It is important to appreciate that although these patients have troponin concentrations within the normal reference range, troponin is not normally distributed and the majority of healthy persons have concentrations $<5 \mathrm{ng} / \mathrm{L}$. If cardiac troponin concentrations are intermediate and increasing within the reference range, then a further

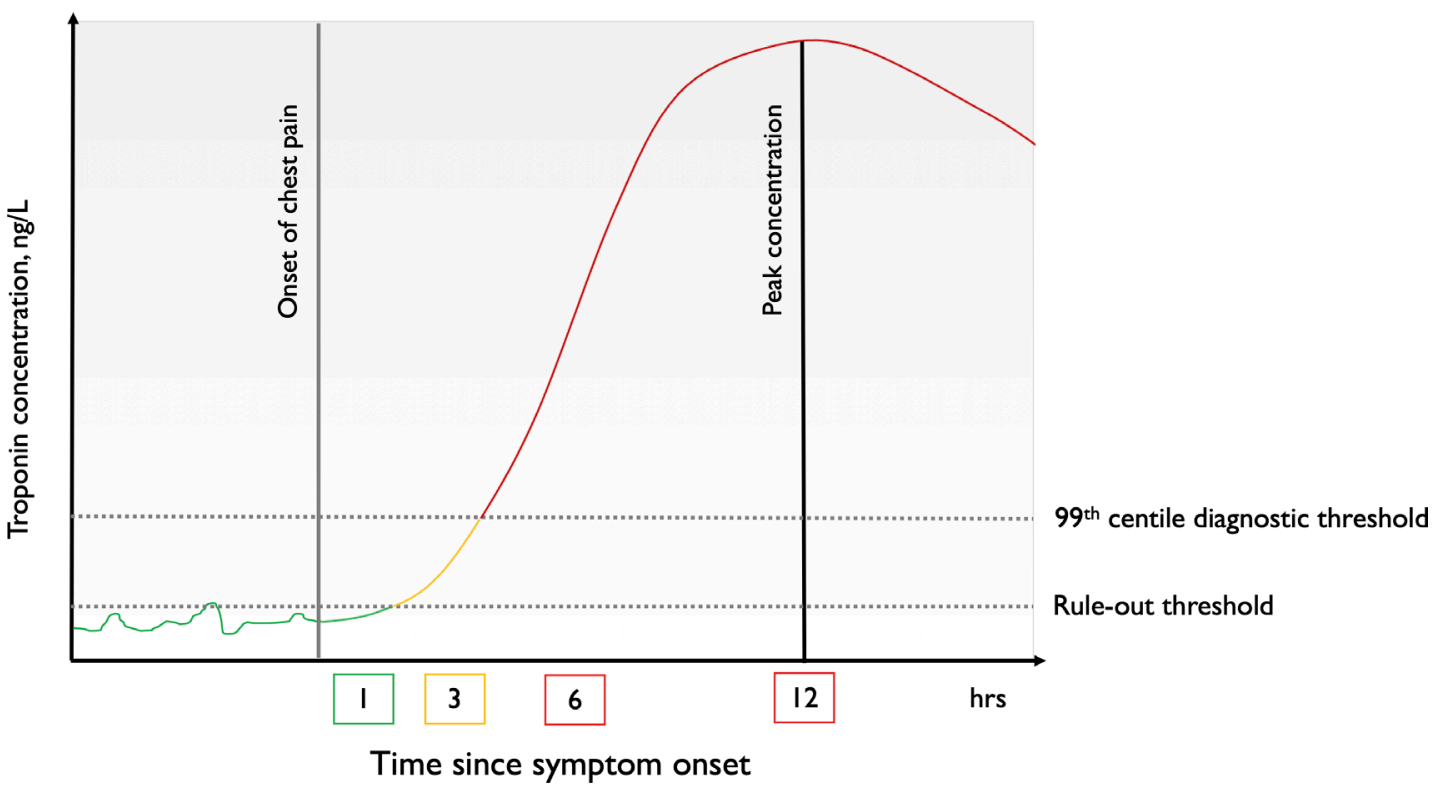

Figure 1 Illustration of cardiac troponin release following the onset of chest pain in acute myocardial infarction. The use of high-sensitivity cardiac troponin assays with separate rule-out and diagnostic thresholds enables earlier clinical decisions. Due to the time delay of troponin detection, patients who present early (within 2 or 3 hours of symptom onset) require serial testing. 


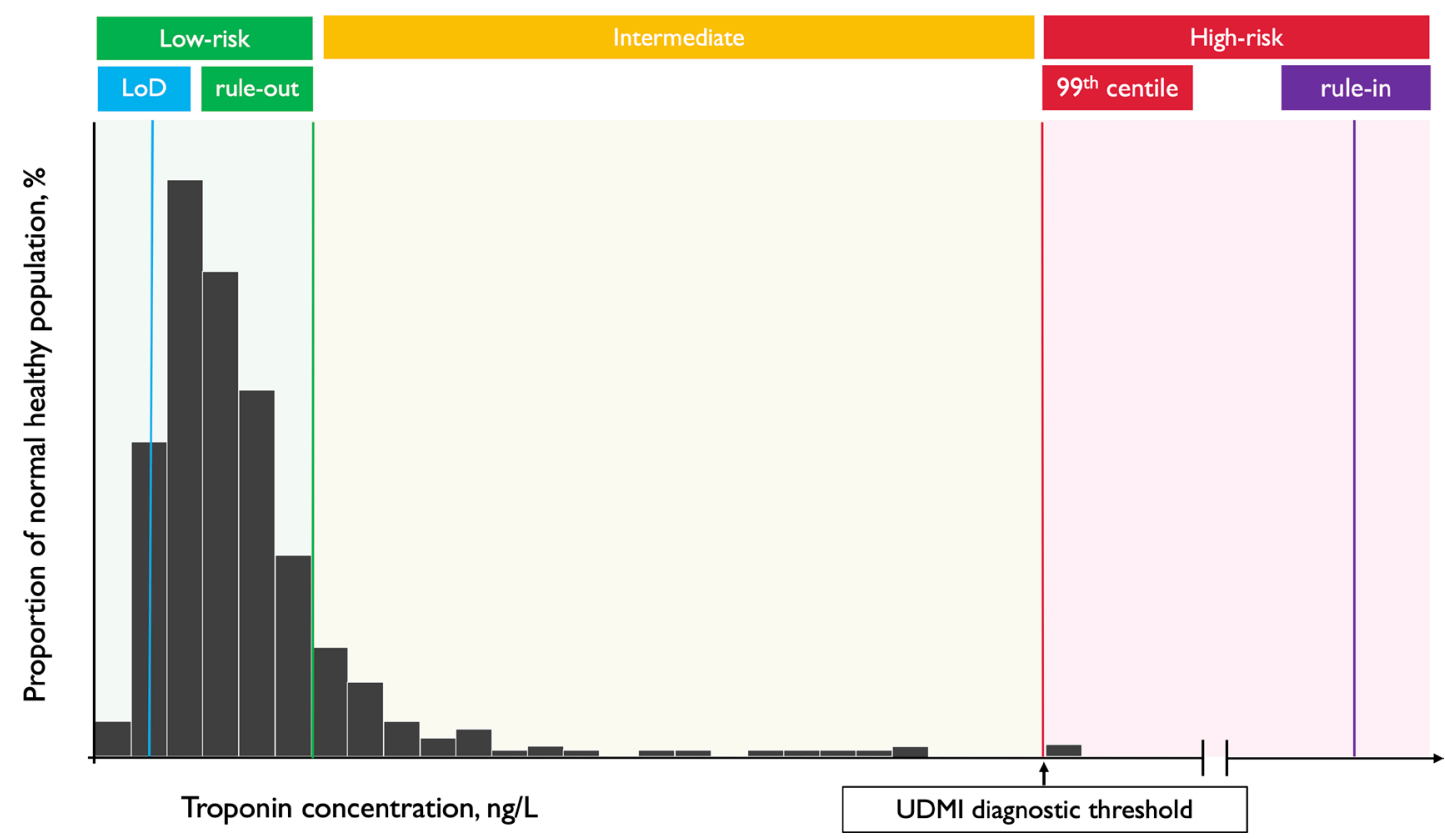

Figure 2 Thresholds to rule out and rule in the diagnosis of myocardial infarction with high-sensitivity cardiac troponin testing. The histogram illustrates the distribution of cardiac troponin in a healthy reference population. High-sensitivity assays can measure cardiac troponin in the majority of the reference population, but concentrations are right-skewed, with the majority of healthy persons having concentrations below the optimised rule-out threshold. The limit of detection (LoD) and optimised thresholds are used to rule out myocardial infarction, and the 99th centile and optimised rule-in thresholds are used to guide further investigations. UDMI, Universal Definition of Myocardial Infarction (the sex-specific 99th centile is the recommended UDMI diagnostic threshold).

measurement 6-12 hours after symptom onset may be required to rule out myocardial infarction. If not, stable intermediate cardiac troponin concentrations may help identify patients with undiagnosed coronary or structural heart disease who do not have myocardial infarction, but who are at increased risk of future cardiac events. ${ }^{23}$ The risk of myocardial infarction or cardiac death at 1 year is five times greater in patients with intermediate cardiac troponin concentrations compared with those below the rule-out threshold $(5.3 \%$ vs $0.7 \%){ }^{19}$

\section{OPTIMISED RULE-IN THRESHOLDS FOR ACUTE MYOCARDIAL INFARCTION}

The specificity and positive predictive value of the 99 th centile are lower in older patients and in those with comorbidities. ${ }^{141524}$ To overcome this optimised rule-in thresholds have been proposed for most hs-cTn assays that aim to provide a consistent positive predictive value of $75 \%$ or more to improve the rapid triage of patients to cardiology. These thresholds differ for each of the hs-cTnT and hs-cTnI assays. ${ }^{2} 182526$

\section{EARLY RULE-OUT PATHWAYS FOR ACUTE MYOCARDIAL INFARCTION}

Based on the principles outlined above, multiple early rule-out pathways have been developed and validated for use in practice. Some have been prospectively evaluated in randomised controlled trials and their effectiveness and safety determined. It is important to remember that these pathways all use hs-cTn assays rather than contemporary sensitive assays, and they should only be applied to patients without ST segment elevation. While there are multiple approaches in the literature, they can be broadly classified into three groups: (1) pathways that rely on the 99th centile diagnostic threshold to both rule in and rule out myocardial infarction; (2) pathways that use separate rule-out and diagnostic thresholds; and (3) pathways that use multiple diagnostic thresholds.

Pathways that use the 99th centile to rule out and rule in myocardial infarction

When hs-cTn assays were first introduced pathways advocating serial testing at presentation and 3 hours later, rather than at 6-12 hours to coincide with peak concentrations, were proposed using the 99th centile to both rule in and rule out myocardial infarction. ${ }^{27} 28$ These pathways were recommended in 2015 by the ESC and by other international guidelines. ${ }^{29}$ However, they were based on retrospective studies where the gold standard was a less sensitive contemporary assay, and it has subsequently been shown in multiple studies that this approach misses 1 in 10 patients with myocardial infarction who would be identified with serial testing 6-12 hours after symptom onset using hs-cTn assay. ${ }^{30-32}$ The diagnostic performance of an approach using a single threshold at the 99th centile can be substantially improved by combining the 99th centile with a validated risk score, such as a history, ECG, age, risk factors, troponin (HEART) score $\leq 3$, thrombolysis in myocardial infarction (TIMI) score $<1$ 
or emergency department assessment of chestpain score $($ EDACS) $<16$, but the proportion of patients ruled out on serial testing is reduced from $70 \%$ using the 99th centile alone to $25 \%, 45 \%$ and $42 \%$, respectively. ${ }^{31}$

\section{Pathways that use separate rule-out and} diagnostic thresholds

Pathways have been developed that use a separate rule-out threshold in combination with the 99th centile diagnostic threshold (figure 3). The HighSTEACS (High-Sensitivity Troponin in the Evaluation of Acute Coronary Syndromes) pathway is an example of this approach. It is based on three basic principles that ensure the pathway performs consistently for hs-cTnI and hs-cTnT assays without modification. First, patients with cardiac troponin concentrations below the rule-out threshold are at very low risk and can be discharged with a single test at presentation. ${ }^{19}$ Second, the pathway is aligned with the universal definition of myocardial infarction and is therefore straightforward to integrate into practice with patients who have cardiac troponin concentrations above the 99th centile diagnostic threshold admitted for serial testing at 6-12 hours and further investigation. Third, patients with intermediate troponin concentrations between the rule-out and diagnostic thresholds require a second test in the emergency department 3 hours from arrival ( 2 hours between tests). A meaningful change in hs-cTn concentration of $\geq 3 \mathrm{ng} / \mathrm{L}$ is based on the lowest measurable change that exceeds the analytical and biological variation of hs-cTn assays. ${ }^{33}$ Those with stable intermediate hs-cTn concentrations can be considered for further investigation as an outpatient, whereas increasing hs-cTn concentrations within the reference range may be important and hospital admission is required for further testing at 6-12 hours. The main advantages of these pathways are their simplicity, and that they enable a decision in all patients and safe discharge of three-quarters of patients, with hospital admission for further testing in a quarter. The main disadvantages are that the diagnostic performance of the 99th centile is lower in older patients with comorbidities and a second test is required 3 hours from arrival in one in five patients with intermediate cardiac troponin concentrations. This can put pressure on quality metrics to reduce crowding in emergency departments, such as the 4-hour target in the UK.

Pathways that use multiple diagnostic thresholds A number of pathways have been proposed that use multiple combinations of thresholds and delta change values to both optimise the rule-out and rule-in of myocardial infarction with serial testing at 1 or 2 hours following the presentation sample (figure 4). This approach aims to identify patients suitable for early discharge from the emergency department as well as those with the highest probability of myocardial infarction who would benefit from early assessment from cardiology services. These pathways were derived in the APACE (Advantageous Predictors of Acute Coronary Syndrome Evaluation) study, a multicentre, prospective, observational cohort study using the hs-cTnT assay. ${ }^{23}$ These pathways use the limit of detection and separate optimised rule-out and rule-in thresholds, with different delta change values to rule out and rule in myocardial infarction. They triage patients to rule-out, observe and rule-in groups. Notably they do not use the 99th centile diagnostic

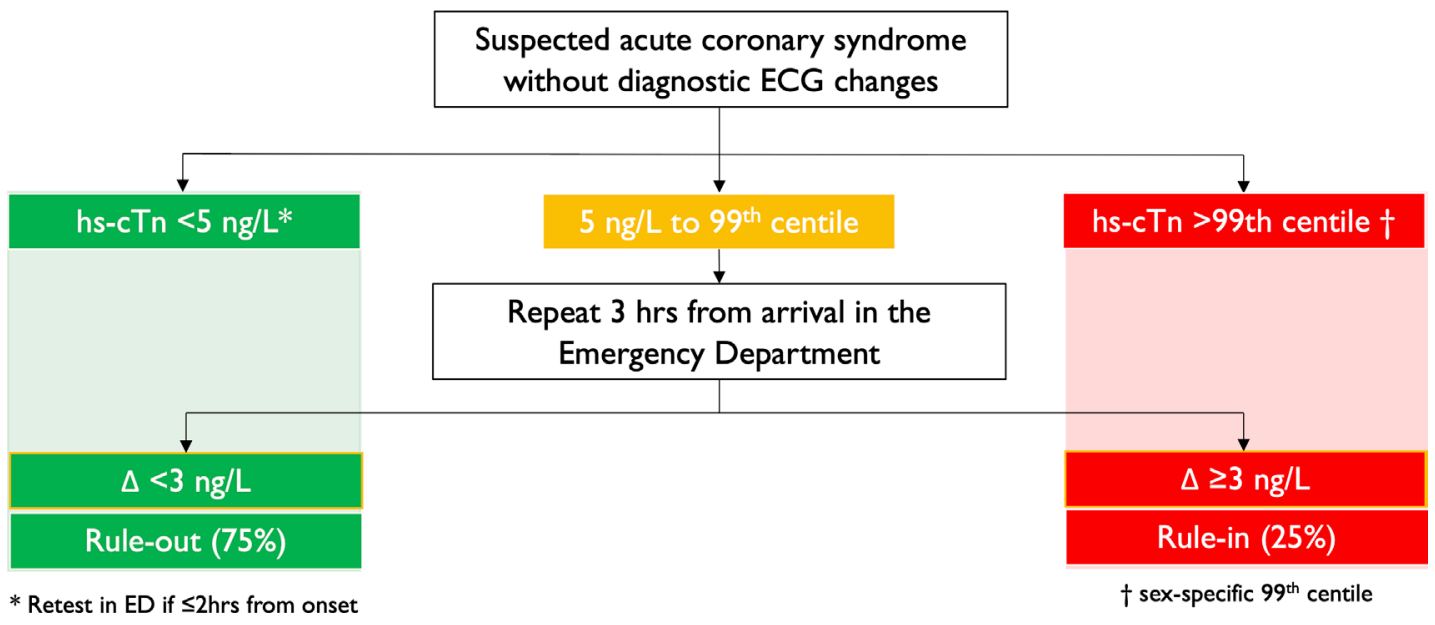

Figure 3 Schematic diagram of an early rule-out pathway using separate rule-out and diagnostic thresholds. The High-STEACS early rule-out pathway is recommended by the ESC and NICE guidelines and uses separate rule-out and diagnostic thresholds with a second test in the ED if needed. Myocardial infarction is ruled out at presentation in patients with hs-cTn (Abbott Diagnostics, Siemens Healthineers, Roche Diagnostics) concentrations $<5 \mathrm{ng} / \mathrm{L}$, unless they presented within 2 hours of symptom onset, where testing is repeated. Patients with cardiac troponin concentrations $\geq 5 \mathrm{ng} / \mathrm{L}$ but below the 99 th centile at presentation are retested in the ED 3 hours after presentation ( 2 hours between samples), and myocardial infarction is ruled out if concentrations are unchanged ( $<3 \mathrm{ng} / \mathrm{L})$ and remain below the 99 th centile diagnostic threshold. A quarter of all patients with suspected acute coronary syndrome have hs-cTn concentrations above the diagnostic threshold or increasing in the reference range, and hospital admission should be considered for further investigation and testing 6-12 hours after symptom onset. ED, emergency department; ESC, European Society of Cardiology; High-STEACS, High-Sensitivity Troponin in the Evaluation of Acute Coronary Syndromes; hs-cTn, high-sensitivity cardiac troponin; NICE, National Institute for Health and Care Excellence. 


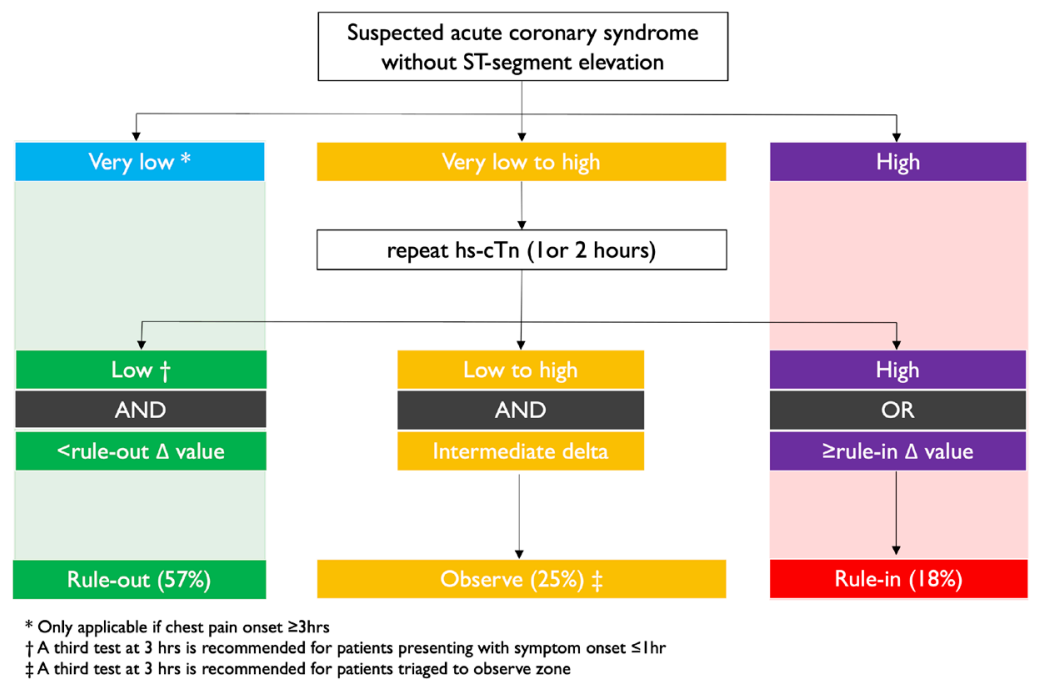

Figure 4 Schematic diagram of an early rule-out pathway using multiple thresholds. This pathway is recommended by the ESC and NICE guidelines and has been validated for multiple hs-cTn assays (Roche Diagnostics, Abbott Diagnostics, Siemens Centaur, Beckman Coulter Access) using six assayspecific thresholds or change values to triage patients into rule-out, observe or rule-in groups. ${ }^{2}$ Myocardial infarction is ruled out if cardiac troponin concentrations are 'very low' (thresholds near or at the limit of detection) in patients presenting at least 3 hours from chest pain onset. Myocardial infarction is ruled in using a single 'high' cardiac troponin threshold at presentation, but serial testing is recommended and the diagnosis based on demonstrating a rise and/or fall in cardiac troponin with one value above the 99th centile diagnostic threshold. Patients with intermediate cardiac troponin concentrations between 'very low' and 'high' should have a second sample 1 or 2 hours after the first test. Myocardial infarction is ruled out at this second step if the repeat concentration is 'low' and there is no significant change using a 'rule-out delta' value. Myocardial infarction is ruled in if cardiac troponin concentrations are now 'high' or there has been an increase in concentrations above the 'rule-in delta' value. It should be noted that these thresholds and delta values differ depending on whether serial testing is performed at 1 or 2 hours. Patients with symptom onset $<3$ hours or intermediate hs-cTn concentrations following the second test between 'low' and 'high' or an intermediate delta change value between the 'rule-out delta' and the 'rule-in delta' are triaged to the observed zone, where a third test 3 hours from the first sample is recommended along with echocardiography. On review of these results clinicians should use clinical judgement to guide further management, and the diagnosis of myocardial infarction is based on documenting a rise and/or fall of hs-cTn with any value above the 99th centile, in line with the universal definition. ESC, European Society of Cardiology; hs-cTn, high-sensitivity cardiac troponin; NICE, National Institute for Health and Care Excellence.

threshold, but rather they incorporate an optimised rule-in threshold that is threefold higher than the 99th centile to identify patients who are more likely to have myocardial infarction. The pathway has since been validated for multiple hs-cTn assays, and users need to familiarise themselves with assay-specific thresholds. $^{23} 2434-36$

The other advantage of these pathways is that the negative predictive value and the positive predictive value of the rule-out and rule-in criteria, respectively, are high in patients who are not triaged to the observe zone, and repeat testing within 1 hour of the initial sample accelerates the diagnostic pathway. The main disadvantage is that the use of six different thresholds to inform clinical decisions is complex and the timing of serial testing needs to be precise as the delta change criteria are dependent on the time interval between tests, which can be challenging to achieve in a busy emergency department. It also remains unclear what the optimal approach to testing and investigation is for patients triaged to the observe zone. Patients in the observe group can have a wide range of hs-cTn values, including intermediate levels below the 99th centile and values greater than the 99 th centile. Patients can also have a delta change that exceeds the analytical and biological variation of hs-cTn assays. Given the wide differential diagnosis, which includes myocardial infarction, patients should be admitted for repeat sampling at 3-6hours. Clinicians should use clinical judgement and consider using clinical risk scores to aid the identification of higher risk patients, with careful consideration given to appropriate monitoring and further investigation.

\section{RANDOMISED CONTROLLED TRIALS OF EARLY RULE-OUT AND RULE-IN PATHWAYS}

The effectiveness and safety of implementing early rule-out and rule-in pathways have been evaluated in three randomised controlled trials to date. The LoDED (Limit of Detection and ECG Discharge) trial compared standard guideline care with a rule-out pathway using the limit of detection of cardiac troponin and a normal ECG in 632 patients. ${ }^{6}$ The use of the LoDED pathway was safe, but did not significantly increase the proportion of patients discharged from hospital within 4 hours of presentation compared with standard care. This surprising finding may have been due to the small sample size and insufficient power or the enrolment of selected lower risk patients. 
The RAPID-TnT (Rapid Assessment of Possible Acute Coronary Syndrome in the Emergency Department With High-Sensitivity Troponin T) study randomised 3378 patients to a 1-hour and 3 -hour rule-out pathway, finding the 1-hour strategy reduced length of stay by $60 \mathrm{~min}$ and increased discharge rates from $32 \%$ to $45 \% .^{5}$ The trial concluded non-inferiority for an endpoint of all-cause mortality or myocardial infarction within 30 days, although this included inpatient events and only one patient had a type 1 myocardial infarction following discharge in each arm of the trial.

The HiSTORIC (High-Sensitivity Cardiac Troponin On Presentation to Rule Out Myocardial Infarction) trial was a stepped wedge, cluster randomised trial that evaluated the efficacy and safety of implementing the High-STEACS early rule-out pathway in 31492 consecutive patients. ${ }^{7}$ The pathway was compared with standard care, which used the 99th centile to rule in and rule out myocardial infarction at presentation in those with more than 6 hours of symptoms or serial testing at presentation and 6-12 hours after symptom onset in those presenting earlier. Introducing the pathway into clinical practice reduced length of stay by 3.3 hours and increased the proportion of patients discharged from $50 \%$ to $71 \%$. Non-inferiority was not formally demonstrated at 30 days, but the observed differences in myocardial infarction or cardiac death following discharge favoured the early rule-out pathway over standard care $(0.3 \%$ (56 of 16792 ) vs $0.4 \%$ (57 of $14700)$ ), and there were no differences in myocardial infarction or cardiac death, hospital reattendance or all-cause mortality at 1 year.

\section{WHAT THE GUIDELINES SAY}

In 2020, NICE published guidance on the use of hs-cTn tests for the early rule-out of non-ST segment elevation myocardial infarction. ${ }^{3}$ The evidence was systematically reviewed, assessed for quality and bias, and clinical and cost-effectiveness were evaluated for all assays and early rule-out pathways that met prespecified minimum diagnostic performance criteria. NICE recommended 11 different hs-cTn assays for use in multiple early rule-out pathways. These include the use of a single sample on presentation using a rule-out threshold at or near the limit of detection, and multiple sample strategies with testing at presentation and $30 \mathrm{~min}$ to 3 hours later if indicated (figures 3 and 4). These pathways were considered safe and cost-effective compared with pathways using the 99th centile diagnostic threshold to both rule in and rule out myocardial infarction or a contemporary sensitive assay.

In 2020, the ESC also published guidance for the management of acute coronary syndromes in patients presenting without persistent ST segment elevation. This guideline also recommended hs-cTn assays over less sensitive tests and that testing should be performed within $60 \mathrm{~min}$ of presentation (level IB). The guideline recommends pathways that use multiple thresholds with accelerated sampling at $0 / 1$ or $0 / 2$ hours (level IB) over the previously recommended 3-hour pathway using the 99th centile to rule in and rule out myocardial infarction. They also recommend the use of validated pathways that use separate rule-out and diagnostic thresholds with testing 3 hours from arrival (level IIa B), such as the High-STEACS pathway (figure 3), as an alternative to this approach.

\section{ISSUES TO CONSIDER WHEN ADOPTING AN EARLY RULE-OUT PATHWAY}

If your hospital is using a contemporary sensitive cardiac troponin assay or a pathway that was developed prior to high-sensitivity testing, then based on the latest randomised clinical trials and guideline recommendations you should consider implementing an hs-cTn assay and a validated early rule-out pathway. Irrespective of the assay or approach there are some practical considerations to remember.

- Involve a multidisciplinary team and prioritise education: Changing the care pathway requires collaboration between laboratory medicine, cardiology and both emergency and acute medicine specialties. Staff need to be aware of the clinical decision thresholds, strengths and limitations of the selected pathway, and follow-up arrangements to be considered for patients now ruled out in the emergency department.

- Know your assay and monitor its performance: Knowledge of your cardiac troponin assay and its performance in routine use is essential. The analytical precision of any laboratory assay can vary, and where clinical decisions are being made based on very low concentrations the performance of the assay at these levels should be monitored regularly using an accredited quality assurance programme.

- ECG takes priority: A 12-lead ECG should be performed within $10 \mathrm{~min}$ of presentation. Patients with ST segment elevation or clear signs of myocardial ischaemia are high risk and care should not be guided by early rule-out pathways. Decision-making using troponin measurements in these patients may delay treatment and is unreliable; one in four patients with ST segment elevation myocardial infarction have cardiac troponin concentrations below the optimised rule-in threshold at presentation as there is no perfusion of the myocardium. ${ }^{37}$

- Early presenters require serial testing: Careful consideration should be given to early presenters as the sensitivity of rule-out thresholds for myocardial infarction is reduced in patients presenting within 2 hours of symptom onset. ${ }^{19}$ In both the HiSTORIC and RAPID-TnT trials serial testing was recommended for patients presenting within 2 and 3 hours of symptom onset, respectively. ${ }^{67}$

- Know when not to incorporate clinical risk scores: Clinical risk scores should be considered to improve the safety of pathways using a single diagnostic threshold to rule in and rule 
out myocardial infarction. However, in early rule-out pathways that use separate rule-out and diagnostic thresholds, no risk score has been shown to improve diagnostic performance and all reduce effectiveness.

- Consider how patients are selected for testing: The diagnostic performance of cardiac troponin is dependent on the patient population in which it is applied and the positive predictive value is substantially lower in patients without chest pain. ${ }^{38}$ Routine testing in unselected patients will increase the identification of non-ischaemic myocardial injury and limit the effectiveness of early rule-out pathways. ${ }^{39}$

- Understand the impact of renal impairment on performance: Cardiac troponin concentrations are often elevated in patients with renal impairment due to non-ischaemic myocardial injury rather than spurious elevation due to renal clearance and predict risk of major adverse cardiac events. Early rule-out pathways are safe in patients with renal impairment, but these pathways appropriately identify a smaller proportion of patients as low risk and suitable for discharge. ${ }^{15} 24$

- Patients in whom myocardial infarction is ruled out require assessment: The exclusion of myocardial infarction does not equate to immediate discharge, nor are diagnostic pathways a substitute for clinical assessment. Alternative diagnoses should be sought and follow-up considered in patients with symptoms suggestive of angina pectoris, particularly in those with stable intermediate cardiac troponin concentrations at risk of future cardiac events.

\section{SUMMARY}

Until recently, the evidence for the implementation of hs-cTn assays and early rule-out pathways for acute myocardial infarction was limited to observational studies where care was not guided by the diagnostic approach in practice. Recent randomised trials have provided important insights into the safety and effectiveness of these pathways in practice. Current diagnostic and practice guidelines recommend a range of assays and validated pathways, and the choice of approach should be informed by local priorities and the multidisciplinary team with a continuing programme of education provided to all users.
Twitter Atul Anand @atula_tweets

Contributors MTHL drafted the manuscript. All authors revised the manuscript critically for important intellectual content and provided their final approval of the version to be published. All authors are accountable for the work.

Funding NLM is supported by the British Heart Foundation through a Butler Senior Clinical Research Fellowship (FS/16/14/32023), Programme Grant (RG/20/10/34966) and a Research Excellence Award (RE/18/5/34216).

Competing interests AA has received honoraria from Abbott Diagnostics. NLM reports research grants awarded to the University of Edinburgh from Abbott Diagnostics and Siemens Healthineers outside the submitted work, and honoraria from Abbott Diagnostics, Siemens Healthineers, Roche Diagnostics and LumiraDx. All other authors have no interests to declare.

Patient and public involvement Patients and/or the public were not involved in the design, or conduct, or reporting, or dissemination plans of this research.

Patient consent for publication Not required.

Provenance and peer review Commissioned; externally peer reviewed.

Author note References which include a * are considered to be key references.

Open access This is an open access article distributed in accordance with the Creative Commons Attribution Non Commercial (CC BY-NC 4.0) license, which permits others to distribute, remix, adapt, build upon this work non-commercially, and license their derivative works on different terms, provided the original work is properly cited, appropriate credit is given, any changes made indicated, and the use is non-commercial. See: http:// creativecommons.org/licenses/by-nc/4.0/.

\section{ORCID iDs}

Matthew T H Lowry http://orcid.org/0000-0001-8972-5136

Atul Anand http://orcid.org/0000-0002-6428-4554

Nicholas L Mills http://orcid.org/0000-0003-0533-7991

\section{REFERENCES}

1 Goodacre S, Cross E, Arnold J, et al. The health care burden of acute chest pain. Heart 2005;91:229-30.

*2 Collet J-P, Thiele H, Barbato E, et al. 2020 ESC guidelines for the management of acute coronary syndromes in patients presenting without persistent ST-segment elevation. Eur Heart J 2021:42:1289-367.

*3 National Institue for Health and Care Excellence. High-sensitivity tests for the early rule-out of NSTEMI [DG40]; 2020.

*4 Apple FS, Sandoval Y, Jaffe AS, et al. Cardiac troponin assays: guide to understanding analytical characteristics and their impact on clinical care. Clin Chem 2017:63:73-81.

*5 Chew DP, Lambrakis K, Blyth A, et al. A randomized trial of a 1hour troponin T protocol in suspected acute coronary syndromes. Circulation 2019;140:1543-56.

*6 Carlton EW, Ingram J, Taylor H, et al. Limit of detection of troponin discharge strategy versus usual care: randomised controlled trial. Heart 2020;106:1586-94.

*7 Anand A, Lee KK, Chapman AR, et al. High-Sensitivity cardiac troponin on presentation to rule out myocardial infarction: a stepped-wedge cluster randomized controlled trial. Circulation 2021;143:2214-24 https://www.ahajournals.org/doi/abs/10. 1161/CIRCULATIONAHA. 120.052380

8 Árnadóttir Ásthildur, Pedersen S, Bo Hasselbalch R, et al. Temporal release of high-sensitivity cardiac troponin $\mathrm{T}$ and I and copeptin after brief induced coronary artery balloon occlusion in humans. Circulation 2021:143:1095-104.

9 Welsh P, Preiss D, Hayward C, et al. Cardiac Troponin $T$ and Troponin I in the general population. Circulation 2019;139:2754-64

10 Thygesen K, Alpert JS, Jaffe AS, et al. Fourth universal definition of myocardial infarction (2018). Eur Heart J 2019;40:237-69.

11 Kimenai DM, Janssen EBNJ, Eggers KM, et al. Sex-specific versus overall clinical decision limits for cardiac troponin I and $\mathrm{T}$ for the diagnosis of acute myocardial infarction: a systematic review. Clin Chem 2018;64:1034-43. 
12 Shah ASV, Ferry AV, Mills NL. Cardiac biomarkers and the diagnosis of myocardial infarction in women. Curr Cardiol Rep 2017:19:40.

13 Shah ASV, Griffiths M, Lee KK, et al. High sensitivity cardiac troponin and the under-diagnosis of myocardial infarction in women: prospective cohort study. BMJ 2015;350:g7873.

14 Eggers KM, Venge P, Lindahl B, et al. Cardiac troponin I levels measured with a high-sensitive assay increase over time and are strong predictors of mortality in an elderly population. J Am Coll Cardiol 2013:61:1906-13.

15 Miller-Hodges E, Anand A, Shah ASV, et al. High-sensitivity cardiac troponin and the risk stratification of patients with renal impairment presenting with suspected acute coronary syndrome Circulation 2018;137:425-35.

16 Chapman AR, Lee KK, McAllister DA, et al. Association of highsensitivity cardiac troponin I concentration with cardiac outcomes in patients with suspected acute coronary syndrome. JAMA 2017;318:1913-24

17 Pickering JW, Than MP, Cullen L, et al. Rapid rule-out of acute myocardial infarction with a single high-sensitivity cardiac troponin T measurement below the limit of detection. Ann Intern Med 2017:166:715-24.

18 Sandoval Y, Nowak R, deFilippi CR, et al. Myocardial infarction risk stratification with a single measurement of high-sensitivity troponin I. J Am Coll Cardiol 2019;74:271-82

19 Bularga A, Lee KK, Stewart S, et al. High-sensitivity troponin and the application of risk stratification thresholds in patients with suspected acute coronary syndrome. Circulation 2019;140:1557-68

20 Blankenberg S, Salomaa V, Makarova N, et al. Troponin I and cardiovascular risk prediction in the general population: the BiomarCaRE Consortium. Eur Heart J 2016;37:2428-37.

21 Body R, Mueller C, Giannitsis E, et al. The use of very low concentrations of high-sensitivity troponin $T$ to rule out acute myocardial infarction using a single blood test. Acad Emerg Med 2016;23:1004-13.

22 Shah ASV, Anand A, Sandoval Y, et al. High-sensitivity cardiac troponin I at presentation in patients with suspected acute coronary syndrome: a cohort study. Lancet 2015:386:2481-8.

23 Neumann JT, Twerenbold R, Ojeda F, et al. Application of highsensitivity troponin in suspected myocardial infarction. N Engl J Med Overseas Ed 2019;380:2529-40.

24 Twerenbold R, Badertscher P, Boeddinghaus J, et al. 0/1-Hour triage algorithm for myocardial infarction in patients with renal dysfunction. Circulation 2018;137:436-51.

25 Reichlin T, Twerenbold R, Wildi K, et al. Prospective validation of a 1-hour algorithm to rule-out and rule-in acute myocardial infarction using a high-sensitivity cardiac troponin T assay. Can Med Assoc J 2015;187:E243-52.
26 Rubini Gimenez M, Twerenbold R, Jaeger C, et al. One-hour rule-in and rule-out of acute myocardial infarction using high-sensitivity cardiac troponin I. Am J Med 2015;128:861-70.

27 Keller T, Zeller T, Ojeda F, et al. Serial changes in highly sensitive troponin I assay and early diagnosis of myocardial infarction. JAMA 2011:306:2684-93.

28 Reichlin T, Hochholzer W, Bassetti S, et al. Early diagnosis of myocardial infarction with sensitive cardiac troponin assays. $N$ Engl J Med Overseas Ed 2009;361:858-67.

29 Roffi M, Patrono C, Collet J-P, et al. 2015 ESC quidelines for the management of acute coronary syndromes in patients presenting without persistent ST-segment elevation. Eur Heart J 2016:37:267-315.

30 Pickering JW, Greenslade JH, Cullen L, et al. Validation of presentation and $3 \mathrm{~h}$ high-sensitivity troponin to rule-in and ruleout acute myocardial infarction. Heart 2016;102:1270-8.

31 Chapman AR, Hesse K, Andrews J, et al. High-Sensitivity cardiac troponin I and clinical risk scores in patients with suspected acute coronary syndrome. Circulation 2018:138:1654-65.

32 Chapman AR, Fujisawa T, Lee KK, et al. Novel high-sensitivity cardiac troponin I assay in patients with suspected acute coronary syndrome. Heart 2019;105:616-22.

33 Kavsak PA, Don-Wauchope AC, Hill SA, et al. Acceptable analytical variation may exceed high-sensitivity cardiac troponin I cutoffs in early rule-out and rule-in acute myocardial infarction algorithms. Clin Chem 2016:62:887-9.

34 Boeddinghaus J, Twerenbold R, Nestelberger T, et al. Clinical validation of a novel high-sensitivity cardiac troponin I assay for early diagnosis of acute myocardial infarction. Clin Chem 2018;64:1347-60.

35 Twerenbold R, Neumann JT, Sörensen NA, et al. Prospective validation of the $0 / 1-h$ algorithm for early diagnosis of myocardial infarction. J Am Coll Cardiol 2018:72:620-32.

36 Mueller C, Giannitsis E, Christ M, et al. Multicenter evaluation of a 0-Hour/1-Hour algorithm in the diagnosis of myocardia infarction with high-sensitivity cardiac troponin T. Ann Emerg Med 2016:68:76-87.

37 Wereski R, Chapman AR, Lee KK, et al. High-Sensitivity cardiac troponin concentrations at presentation in patients with ST-segment elevation myocardial infarction. JAMA Cardiology 2020;5:1302.

38 Lee KK, Noaman A, Vaswani A, et al. Prevalence, determinants, and clinical associations of high-sensitivity cardiac troponin in patients attending emergency departments. Am J Med 2019:132:110. e8-110.e21.

39 Shah ASV, Sandoval Y, Noaman A, et al. Patient selection for high sensitivity cardiac troponin testing and diagnosis of myocardial infarction: prospective cohort study. BMJ 2017;359:j4788 